\title{
ERGODIC EQUIVALENCE RELATIONS, COHOMOLOGY, AND VON NEUMANN ALGEBRAS
}

\author{
BY JACOB FELDMAN ${ }^{1}$ AND CALVIN C. MOORE ${ }^{2}$
}

Communicated April 3, 1975

1. Introduction. Throughout, $(X, B)$ will be a standard Borel space, $G$ some countable group of automorphisms, $R_{G}$ the equivalence relation $\{(x, g \cdot x), g \in G\}$, and $\mu$ a $\sigma$-finite measure on $X$. For $\mu$ quasi-invariant, the orbit structure of the action has been studied by Dye [4] , [5], Krieger [8]- [13], and others. Here, ignoring $G$ and focusing on $R_{G}$ via an axiomatization, and studying a cohomology for $R_{G}$, we obtain a variety of results about group actions and von Neumann algebras. The major results are stated below.

2. Equivalence relations. $R$ will be an equivalence relation on $X$ with all equivalence classes countable, and $R \in B \times B$.

THEOREM 1. Every $R$ is an $R_{G}$.

Properties of $G$-actions translate into properties of $R_{G}$ which can be stated with no $G$ in sight, e.g., quasi-invariance, ergodicity. Let $\mu$ be quasi-invariant, and let $C=B \times\left. B\right|_{R}$ and $P_{l}(x, y)=x, P_{r}(x, y)=y$. Now $C$ has a natural measure class as follows:

THEOREM 2. The formula $\nu_{l}(C)=\int\left|P_{l}^{-1}(x) \cap C\right| d \mu(x)$, where $|\cdot|$ is cardinality, and a similar formula for $\nu_{r}$ define equivalent $\sigma$-finite measures on $C$.

The Radon-Nikodym derivative is the function $D=d \nu_{r} / d \nu_{l}$ on $R$. If $R=R_{G}$, then $d(\mu \cdot g) / d \mu(x)=D(x, g x)$. Moreover, $D$ is a cocycle in that $D(x, y) D(y, z)$ $=D(x, z)$ a.e. and the $D^{\prime}$ arising from a $\mu^{\prime}$ equivalent to $\mu$ is cohomologous to $D$.

For ergodic $R$, one has a classification into types which are $\mathrm{I}_{n}, n=1, \ldots$, $\infty, \mathrm{II}_{1}, \mathrm{II}_{\infty}$ and III as in [3]. For $j=1,2$, relations $R_{j}$ on $\left(X_{j}, B_{j}, \mu_{j}\right)$ are isomorphic if there is a Borel isomorphism $a: X_{1} \rightarrow X_{2}$ with $\mu \sim \mu \circ a^{-1}$ and $R_{2}(a(x))$ $=a\left(R_{1}(x)\right)$ a.e. If the $R_{j}$ are ergodic, they are principal groupoids and, hence, define virtual groups [14].

THEOREM 3. $R_{1}$ and $R_{2}$ define isomorphic virtual groups iff each is isomorphic to a restriction of the other, where the restriction of $R$ to $H$ is $R \cap H \times H$. Hence, the two notions of isomorphism coincide if $R_{1}$ and $R_{2}$ are both of infinite type.

Hyperfiniteness in terms of $R$ becomes: $\exists R_{n} \uparrow R$ with $\left|R_{n}(x)\right|$ finite $\forall n, \forall x$.

AMS (MOS) subject classifications (1970). Primary 22D25, 22D40, 28A65, 46L10.

1 Supported in part by National Science Foundation Grant MPS-75-05576.

2 Supported in part by National Science Foundation Grant MPS-74-19876. 
3. Cohomology. For simplicity assume that $R$ is ergodic and let $R^{n}=\left\{\left(x_{0}\right.\right.$, $\left.\left.\ldots, x_{n}\right), x_{0} \sim \cdots \sim x_{n}\right\} \subset X^{n+1}$ with the natural measure class generalizing that of Theorem 2. An $R$ module is an abelian polonais group with a Borel map $u$ of $R$ into $\operatorname{Aut}(A)$ with $u(x, y) u(y, z)=u(x, z)$. Define cochain groups $C^{n}(R, A)$ as the Borel functions mod null functions from $R^{n}$ to $A$ with coboundary operators $\left(\delta_{n} c\right)\left(x_{0}, \ldots, x_{n+1}\right)=\Sigma_{j}(-1)^{j} c\left(x_{0}, \ldots, \hat{x}_{j}, \ldots, x_{n}\right)$ if $u=1$ and with a slight modification if $u \neq 1$. We define cohomology groups $H^{n}(G, A)$ of this complex; also for $n=1$, we allow $A$ to be nonabelian and obtain a cohomology set. These groups were introduced in the virtual group context by Westman [17]. We show how to axiomatize these groups and show that they are unique solutions to a universal problem. If $R \sim R_{G}$ with $G$ acting freely, one may identify $H^{n}(R, A)$ with $H^{n}(G, U(X, A))$, where $U(X, A)$ is Borel functions mod null functions from $X$ to $A$ with $G$ operating suitably. If $R$ is hyperfinite and not type $\mathrm{I}_{n}$ so that $R=R_{Z}$, with $Z$ acting freely, then $H^{n}(R, A)=0$ for all $n \geqslant 2$. Since any action of an abelian group is hyperfinite (Dye [5], Feldman and Lind [6]), one can obtain results of the following kind:

THEOREM 4. If $s$ and $t$ are commuting ergodic independent $\left(s^{n} \neq t^{m}\right)$ automorphisms of $(X, \mu)$, then for any Borel function $f$ from $X$ to the circle $T$, there exist Borel functions $g$ and $h$ to $T$ so that $f=((g \circ s) / g)((h \circ t) / h)$ a.e.

Generalizing Mackey [14], we define for $c \in Z^{1}(R, A)$ a relation $R(c)$ on $X \times A$ by $(x, a) \sim\left(x^{1}, a^{1}\right)$ iff $x \sim x^{1}$ and $c\left(x, x^{1}\right) a=a^{1}$, where $A$ is an abelian locally compact $R$ module with trivial action. Then $A$ acts by right translations on $X \times A$ and preserves $R(c)$ and so acts via Mackey's point realization theorem on $Z=X \times A / \widetilde{R(c)}$, where $\widetilde{R(c)}$ is a countably separated equivalence relation containing $R(c)$ whose image in the measure algebra of $X \times A$ coincides with the $R(c)$ invariant sets. This ergodic action of $A$ is called the range of $c$, and depends only on the class of $c$. The isotropy group $A_{z}$ of $A$ at $z \in Z$ is an a.e. constant closed subgroup $A(c)$ which is called the proper range of $c$. Now if $A^{*}$ is the one point compactification of $A$, we generalize [12] and define the asymptotic range $r_{\infty}^{*}(c)$ as the intersection over all subsets $B$ of $X$ of positive measure of the essential ranges in $A^{*}$ of $c$ restricted to $B \times B$, and $r^{*}(c)=r_{\infty}^{*}(c) \cap A$. An important result is

THEOREM 5. $r^{*}(c)$ is a closed subgroup of $A$ depending only on the class of $c$ and equals the proper range $A(c)$ of $c$.

For $A=R$ and $c=\log D$, this was done by enumeration of cases in [7], and there is some overlap with results in [2]. We also have

THEOREM 6. For $A=\mathbf{R}^{n}+\mathbf{Z}^{m}, c \sim 0$ iff $\infty \notin r_{\infty}^{*}(c)$.

As a corollary we obtain the result that if $\log D$ is bounded, then there is an equivalent invariant measure, a result that also follows from Theorem 1 and [15]. 
4. von Neumann algebras. Generalizing the Zeller-Meier generalization [18] of the Murray-von Neumann group measure space factors, we construct for an ergodic relation $R$ and $t \in H^{2}(R, T)$ a factor $M(R, t)$ which we view as the "twisted algebra of matrices over $R$ ". For $t=1$ this factor is constructed (less transparently) in [10]. Our Hilbert space $H$ is $L_{2}\left(R, \nu_{l}\right)$, and we pick $c \in t$ normalized to be skew symmetric. For $F \in L^{\infty}(R)$ which is band limited in that $\mid\{x \mid F(x, y) \neq 0$ and $0 \neq F(y, x)\} \mid$ is bounded, one defines an operator $M_{F}$ on $H$ by

$$
\left(M_{F} f\right)(x, z)=\sum_{y \sim x} f(x, y) F(y, z) c(x, y, z) .
$$

These operators form a $*$-algebra whose weak closure is a factor $M(R, t)$ depending only on $t$ and not on $c$. The commutant has a similar form. The indicator function of the diagonal $\Delta$ is a separating and cyclic vector, and the diagonal subalgebra $A=\left\{M_{F}, F=0\right.$ off $\left.\Delta\right\}$ is a maximal abelian subalgebra which is regular by Theorem 1. Moreover, there is a normal faithful conditional expectation $E$ of $M(R, t)$ onto $A$. If $M$ is any factor with abelian subalgebra $A$ satisfying these conditions, we call $A$ a Cartan subalgebra [19]. One of our major results is a converse of this construction.

THEOREM 7. If $A$ is a Cartan subalgebra of the factor $M$, then $M=M(R, t)$ for suitable $R$ and $t$ with $A$ as diagonal subalgebra for any $R^{\prime}$.

Of course, if $M$ is a finite factor, the $E$ always exists. One may ask if $M(R, t)$ determines $R$ and $t$. If we restrict to hyperfinite $R$ (where $t=1$ automatically), then $M(R, 1)$ does indeed determine $R$ by [4], [5], [6]. A major open problem is whether we get all factors as $M(R, t)$ 's. We note that Connes [1] constructs an $M(R, t)$ which is not an $M\left(R^{\prime}, 1\right)$.

Our final results concern automorphisms and conjugacy questions. If $A$ is the diagonal subalgebra of $M=M(R, t)$, let $\operatorname{Out}(M, A)$ be the subgroup of the “outer" automorphism group of $M$ which maps $A$ into something inner conjugate to $A$. Let $\operatorname{Out}(R, t)$ be the group of "outer" automorphisms of the relation $R$ fixing the cohomology class $t$. We have a structure theorem for $\operatorname{Out}(M, A)$ generalizing results in [16].

THEOREM 8. We have an exact sequence $1 \rightarrow H^{1}(R, T) \rightarrow \operatorname{Out}(M, A) \rightarrow$ $\operatorname{Out}(R, t) \rightarrow 1$.

Finally, let $A_{i}$ be two Cartan subalgebras of $M$ with conditional expectations $E_{i}$. The restriction of $E_{1}$ to $A_{2}$ gives rise to a unique positive measure $\gamma$ on $X_{1} \times X_{2}$ (where $A_{i}=L^{\infty}\left(X_{i}, \mu_{i}\right)$ ) whose disintegration products $\gamma_{x}\left(x \in X_{1}\right)$ with respect to projection to $X_{1}$ are determined by $E_{1}(a)(x)=\int a(y) d \gamma_{x}(y)$ a.e. for $a \in A_{2}=L^{\infty}\left(X_{2}, \mu_{2}\right)$. Let us say that $A_{2}$ is discrete over $A_{1}$ if a.a. $\gamma_{x}$ are atomic measures.

THEOREM 9. If $M$ is an infinite factor, $A_{1}$ and $A_{2}$ are inner conjugate iff $A_{2}$ is discrete over $A_{1}$ and $A_{1}$ is discrete over $A_{2}$. 
ADDED IN PROOF. Theorem 5, for hyperfinite $R$, was also obtained by $\mathrm{K}$. Schmidt (Cohomology and skew products of ergodic transformations, University of Warwick, Coventry, England, preprint).

\section{REFERENCES}

1. A. Connes, $\boldsymbol{A}$ factor not antiisomorphic to itself, Queens Mathematical Reprint \#1974-26.

2. A. Connes and M. Takesaki, Flot des poids sur les facteurs de type III, C. R. Acad. Sci. Paris Sér. A-B 278 (1974), A945-A948.

3. Dang-Ngoc-Nghiem, On the classification of dynamical systems, Ann. Inst. Poincare 9 (1973), 397-425.

4. H. A. Dye, On groups of measure preserving transformations. I, Amer. J. Math. 81 (1959), 119-159. MR 24 \#A1366.

5. - On groups of measure preserving transformations. II, Amer. J. Math. 85 (1963), 551-576. MR 28 \#1275.

6. J. Feldman and D. Lind, Hyperfiniteness and the Halmos-Rokhlin theorem for abelian group actions, Proc. Amer. Math. Soc. (to appear).

7. T. Hamachi, Y. Oka and M. Osikawa, Flows associated with ergodic non-singular transformation groups (preprint).

8. W. A. Krieger, On non-singular transformations of a measure space. I, Z. Wahrscheinlichkeitstheorie und Verw. Gebiete 11 (1969), 83-97. MR 39 \#1628.

9. - On non-singular transformations of a measure space. II, Z. Wahrscheinlichkeitstheorie und Verw. Gebiete 11 (1969), 98-119. MR 39 \#1628.

10. - On constructing non-*-isomorphic hyperfinite factors of type III, J. Functional Analysis 6 (1970), 97-109. MR 41 \#4260.

11. - On a class of hyperfinite factors arising from null-recurrent Markov chains, J. Functional Analysis 7 (1971), 27-42. MR 43 \#938.

12. - On the Araki-Woods asymptotic ratio set and nonsingular transformations, Lecture Notes in Math., vol. 160, Springer-Verlag, New York, 1970, pp. 158-177.

13. - On ergodic flows and the isomorphism of factors (preprint).

14. G. W. Mackey, Ergodic theory, and virtual groups, Math. Ann. 166 (1966), 187207. MR $34 \# 1444$.

15. J. M. Rosenblatt, Equivalent invariant measures, Israel J. Math. 17 (1974), 261 270.

16. I. M. Singer, Automorphisms of finite factors, Amer. J. Math. 77 (1955), 117133. MR 16, 597.

17. J. J. Westman, Cohomology for the ergodic actions of countable groups, Proc.

Amer. Math. Soc. 30 (1971), 318-320. MR 43 \#6402.

18. G. Zeller-Meier, Produits croisés $d$ 'une $C^{*}$-algèbre par un groupe d'automorphismes, J. Math. Pures Appl. (9) 47 (1968), 101-239. MR 39 \#3329.

19. A. M. Veršik, Nonmeasurable decompositions, orbit theory, algebras of operators, Dokl. Akad. Nauk SSSR 199 (1971), 1004-1007 = Soviet Math. Dokl. 12 (1971), 12181222. MR $44 \# 4537$.

DEPARTMENT OF MATHEMATICS, UNIVERSITY OF CALIFORNIA, BERKELEY, CALIFORNIA 94720 